\title{
ON HEAT TRANSFER TO PULSATILE FLOW OF A TWO-PHASE FLUID
}

\author{
A. K. GHOSH AND S. P. CHAKRABORTY
}

Received 10 July 2004

The problem of heat transfer to pulsatile flow of a two-phase fluid-particle system contained in a channel bounded by two infinitely long rigid impervious parallel walls has been studied in this paper. The solutions for the steady and the fluctuating temperature distributions are obtained. The rates of heat transfer at the walls are also calculated. The results are discussed numerically with graphical presentations. It is shown that the presence of the particles not only diminishes the steady and unsteady temperature fields but also decreases the reversal of heat flux at the hotter wall irrespective of the influences of other flow parameters.

\section{Introduction}

The problems of heat transfer to fluid flow systems in pipes and channels are particularly important to understand various aspects of transpiration cooling and gaseous diffusion. The exact solutions of some problems associated with heat transfer in an incompressible viscous fluid have already been reported by Schlichting [5]. It has been noticed that the generation of heat due to friction and the variation of pressure gradient usually exert a large effect on the process of cooling and these factors often make the warmer wall heated instead of being cooled.

In recent years, considerable attention has been given to the study of the problems of heat transfer to pulsatile flow of fluids in channels of various cross-sections due to their increasing applications in the analysis of blood flow and in the flows of other biological fluids. Radhakrishnamacharya and Maity [3] have made an investigation of heat transfer to pulsatile flow of a Newtonian viscous fluid in a channel bounded by two infinitely long parallel porous walls with a view to its application in the dialysis of blood in artificial kidneys. This analysis was carried out to determine theoretically the steady and the fluctuating temperature fields and the rates of heat transfer at the walls. It was shown that the rate of heat transfer at the injection wall which was maintained at temperature $T_{0}$ increases with the increase of Eckert number $E_{c}$ while at the suction wall which was kept at temperature $T_{1}\left(>T_{0}\right)$, heat flows from the fluid to the wall even if $T_{1}>T_{0}$. 
On the other hand, a similar problem of heat transfer to pulsatile flow of a viscoelastic fluid in a channel bounded by two infinitely long impervious rigid parallel walls was studied by Ghosh and Debnath [1] with a view to its application in the analysis of blood flow where it is assumed that blood behaves as a viscoelastic fluid in some parts of the vascular channel. This analysis provides theoretical results for the steady and the unsteady temperature fields and the rates of heat transfer at the walls. The effects of large pressure gradient and the elastic parameters on the process of heat transfer have been discussed numerically.

The objective of the present paper is to construct solution of the problem of heat transfer associated with the pulsatile flow of a two-phase fluid-particle system in a channel bounded by two infinitely long impervious rigid parallel walls seperated by a distance $h$ with a view to its more realistic application in the analysis of blood flow in arteries. The analysis is aimed at finding the analytical solutions for the temperature fields for both the fluid and the particles. The rates of heat transfer at the walls are also calculated. The results are discussed numerically through graphical representations. It is shown that the particles have diminishing effects on both the steady and nonsteady temperarture fields of the fluid and the reversal of heat flux at the hotter wall decreases with the increase of particles irrespective of the influences of other flow parameters.

\section{Mathematical formulation}

We consider an unsteady flow of an incompressible viscous fluid with uniformly distributed small inert spherical particles in a channel bounded by two infinitely long impervious rigid parallel walls at a distance $h$ apart which is driven by the pressure gradient of the form

$$
-\frac{1}{\rho} \frac{\partial p}{\partial x}=A\left(1+\epsilon e^{i \omega t}\right)
$$

where $A$ is a known constant, $\epsilon$ is an arbitrarily chosen small positive quantity, and $\omega$ is the frequency.

The flow takes place parallel to the $x$-axis which is taken along the lower wall at $y=0$ and the $y$-axis is normal to the wall. The lower wall at $y=0$ and the upper wall at $y=h$ are maintained at constant temperature $T_{0}$ and $T_{1}\left(T_{1}>T_{0}\right)$, respectively. Following Saffman [4] and Marble [2] the equations governing the motion of the fluid and the particles are given by

$$
\begin{gathered}
\frac{\partial u}{\partial t}=-\frac{1}{\rho} \frac{\partial p}{\partial x}+\nu \frac{\partial^{2} u}{\partial y^{2}}+\frac{k}{\tau_{u}}\left(u_{p}-u\right), \\
\frac{\partial u_{p}}{\partial t}=\frac{1}{\tau_{u}}\left(u-u_{p}\right),
\end{gathered}
$$

where $u$ and $u_{p}$ are respectively the fluid velocity and the particle velocity in the $x$ direction. $\tau_{u}$ is the velocity relaxation time of the particles which represents the time scale on which the particle velocity adjusts to changes in the surrounding fluid velocity and 
$k=\rho_{p} / \rho$ represents the ratio of mass density of the particles and the fluid density is usually termed as mass concentration of the particles.

The energy equations for the respective phases may be written as

$$
\begin{gathered}
\frac{\partial T}{\partial t}=\frac{k}{\tau_{T}}\left(T_{p}-T\right)+\frac{\chi}{\rho C_{p}} \frac{\partial^{2} T}{\partial y^{2}}+\frac{\mu}{\rho C_{p}}\left(\frac{\partial u}{\partial y}\right)^{2}+\frac{k}{C_{p} \tau_{u}}\left(u_{p}-u\right)^{2}, \\
\frac{\partial T_{p}}{\partial t}=\frac{1}{\tau_{T}}\left(T-T_{p}\right),
\end{gathered}
$$

where $C_{p}, \chi, \mu$ are respectively the specific heat, the thermal conductivity, and the coefficient of dynamic viscosity. $T$ and $T_{p}$ are the temperature fields respectively for the fluid and the particle phases. $\tau_{T}$ is the thermal relaxation time of the particles similar in meaning to that of $\tau_{u}$.

Equations (2.2) to (2.5) are to be solved subject to the conditions

$$
\begin{gathered}
u=0, \quad T=T_{0} \quad \text { at } y=0, \\
u=0, \quad T=T_{1} \quad \text { at } y=h, T_{1}>T_{0} .
\end{gathered}
$$

We now consider the following dimensionless flow variables and the flow parameters

$$
\begin{gathered}
u^{*}, u_{p}^{*}=\frac{u, u_{p}}{A h^{2} / v}, \quad z=\frac{y}{h}, \quad t^{*}=\frac{t v}{h^{2}}, \quad \sigma=\frac{\omega h^{2}}{\nu}, \\
\left(\lambda_{1}, \lambda_{2}\right)=\frac{\left(\tau_{u}, \tau_{T}\right) v}{h^{2}}, \quad \theta=\frac{T-T_{0}}{T_{1}-T_{0}}, \quad P_{r}=\frac{\mu C_{p}}{\chi}, \quad E_{c}=\frac{A^{2} h^{4}}{v^{2} C_{p}\left(T_{1}-T_{0}\right)},
\end{gathered}
$$

where $\nu=\mu / \rho$ is the coefficient of kinematic viscosity, $P_{r}$ is Prandtl number, and $E_{c}$ is the Eckert number.

Introducing the nondimensional quantities given in (2.7) in the equations (2.2) to (2.5) together with (2.1), we get

$$
\begin{gathered}
\frac{\partial u^{*}}{\partial t^{*}}=1+\epsilon e^{i \sigma t^{*}}+\frac{\partial^{2} u^{*}}{\partial z^{2}}+\frac{k}{\lambda_{1}}\left(u_{p}^{*}-u^{*}\right), \\
\frac{\partial u_{p}^{*}}{\partial t^{*}}=\frac{1}{\lambda_{1}}\left(u^{*}-u_{p}^{*}\right), \\
\frac{\partial \theta}{\partial t^{*}}=\frac{k}{\lambda_{2}}\left(\theta_{p}-\theta\right)+\frac{1}{P_{r}} \frac{\partial^{2} \theta}{\partial z^{2}}+E_{c}\left(\frac{\partial u^{*}}{\partial z}\right)^{2}+\frac{k E_{c}}{\lambda_{1}}\left(u_{p}^{*}-u^{*}\right)^{2}, \\
\frac{\partial \theta_{p}}{\partial t^{*}}=\frac{1}{\lambda_{2}}\left(\theta-\theta_{p}\right) .
\end{gathered}
$$


2500 On heat transfer to pulsatile flow of a two-phase fluid

These equations are to be solved subject to the conditions

$$
\begin{array}{rlrl}
u^{*}=0 & \text { at } z=0,1, \\
\theta=0 & \text { at } z=0, \\
& =1 & & \text { at } z=1 .
\end{array}
$$

\section{Solution for the velocity field}

We assume

$$
\begin{gathered}
u^{*}=u_{0}+\epsilon u_{1} e^{i \sigma t^{*}}, \\
u_{p}^{*}=u_{p 0}+\epsilon u_{p 1} e^{i \sigma t^{*}} .
\end{gathered}
$$

Introducing (3.1) in (2.8) and (2.9) and solving them with the help of (2.12), we get

$$
\begin{gathered}
u_{0}=u_{p 0}=\frac{1}{2} z(1-z), \\
u_{1}=\left(1+i \sigma \lambda_{1}\right) u_{p 1}=\frac{1}{M^{2}}\left[1-\frac{\sinh M(1-z)+\sinh M z}{\sinh M}\right],
\end{gathered}
$$

where

$$
M^{2}=\frac{i \sigma\left(1+k+i \sigma \lambda_{1}\right)}{1+i \sigma \lambda_{1}} .
$$

Thus (3.1) together with (3.2) and (3.3) constitute the solution for the velocity field $u^{*}$ and $u_{p}^{*}$ of the fluid and the particles, respectively. The shear stress at the walls is given by

$$
\begin{gathered}
\tau_{0}^{*}=\frac{\tau_{0}}{\rho A}=\left(\frac{\partial u^{*}}{\partial z}\right)_{z=0}=\frac{1}{2}+\epsilon\left|M_{0}\right| e^{i\left(\sigma t^{*}+\alpha_{0}\right)}, \\
\tau_{1}^{*}=\frac{\tau_{1}}{\rho A}=\left(\frac{\partial u^{*}}{\partial z}\right)_{z=1}=-\frac{1}{2}-\epsilon\left|M_{0}\right| e^{i\left(\sigma t^{*}+\alpha_{0}\right)},
\end{gathered}
$$

where

$$
\begin{gathered}
M_{0}=\frac{1}{M} \tanh \frac{M}{2}, \\
\left|M_{0}\right|=\left[\frac{\cosh m_{r}-\cos m_{i}}{\left(m_{r}^{2}+m_{i}^{2}\right)\left(\cosh m_{r}+\cos m_{i}\right)}\right]^{1 / 2}, \\
\alpha_{0}=\tan ^{-1}\left(\frac{m_{r} \sin m_{i}-m_{i} \sinh m_{r}}{m_{r} \sinh m_{r}+m_{i} \sin m_{i}}\right), \\
M=\left(m_{r}, m_{i}\right)=\left[\frac{\sigma}{2\left(1+\sigma^{2} \lambda_{1}^{2}\right)}\left\{\left[k^{2} \sigma^{2} \lambda_{1}^{2}+\left(1+k+\sigma^{2} \lambda_{1}^{2}\right)^{2}\right]^{1 / 2} \pm k \sigma \lambda_{1}\right\}\right]^{1 / 2} .
\end{gathered}
$$


Since $m_{r}, m_{i}$ are both positive and $\alpha_{0}$ is negative, it is evident from (3.7) and (3.8) that the presence of particles decreases both the magnitude of the skin friction fluctuation and the phase lag at the walls.

\section{Solution for the temperature field}

Since $u^{*}, u_{p}^{*}$ in $(3.1)$ are real, $u^{*}, u_{p}^{*}$ can be written in more convenient form as

$$
\begin{gathered}
u^{*}=u_{0}+\frac{\epsilon}{2}\left[u_{1} e^{i \sigma t^{*}}+\bar{u}_{1} e^{-i \sigma t^{*}}\right], \\
u_{p}^{*}=u_{p 0}+\frac{\epsilon}{2}\left[u_{p 1} e^{i \sigma t^{*}}+\bar{u}_{p 1} e^{-i \sigma t^{*}}\right] .
\end{gathered}
$$

This leads us to assume the temperature field as

$$
\begin{gathered}
\theta=\theta_{0}+\frac{\epsilon}{2}\left[\theta_{1} e^{i \sigma t^{*}}+\bar{\theta}_{1} e^{-i \sigma t^{*}}\right]+\frac{\epsilon^{2}}{2}\left[\theta_{2} e^{2 i \sigma t^{*}}+\bar{\theta}_{2} e^{-2 i \sigma t^{*}}\right] \\
\theta_{p}=\theta_{p 0}+\frac{\epsilon}{2}\left[\theta_{p 1} e^{i \sigma t^{*}}+\bar{\theta}_{p 1} e^{-i \sigma t^{*}}\right]+\frac{\epsilon^{2}}{2}\left[\theta_{p 2} e^{2 i \sigma t^{*}}+\bar{\theta}_{p 2} e^{-2 i \sigma t^{*}}\right] .
\end{gathered}
$$

Introducing (4.1) to (4.4) in equations (2.10) and (2.11) and equating terms independent of $t$ and the coefficients of $e^{i \sigma t^{*}}$ and $e^{2 i \sigma t^{*}}$, we obtain the following set of equations for the determination of $\theta_{0}, \theta_{1}, \theta_{2}$ and $\theta_{p 0}, \theta_{p 1}, \theta_{p 2}$. These equations with appropiate boundary conditions are

$$
\begin{gathered}
\frac{\partial^{2} \theta_{0}}{\partial z^{2}}=-E_{c} P_{r}\left[\left(\frac{\partial u_{0}}{\partial z}\right)^{2}+\frac{\epsilon^{2}}{2}\left\{\frac{\partial u_{1}}{\partial z} \frac{\partial \bar{u}_{1}}{\partial z}+\frac{k \sigma^{2} \lambda_{1}}{1+\sigma^{2} \lambda_{1}^{2}} u_{1} \bar{u}_{1}\right\}\right], \\
\theta_{p 0}=\theta_{0}, \quad \theta_{0}=(0,1) \quad \text { at } z=(0,1), \\
\frac{\partial^{2} \theta_{1}}{\partial z^{2}}-P_{r} N_{1}^{2} \theta_{1}=-2 E_{c} P_{r} \frac{\partial u_{0}}{\partial z} \frac{\partial u_{1}}{\partial z}, \\
\theta_{p 1}=\frac{\theta_{1}}{1+i \sigma \lambda_{2}}, \quad \theta_{1}=(0,0) \quad \text { at } z=(0,1), \\
\frac{\partial_{1}^{2} \theta_{2}}{\partial z^{2}}-2 P_{r} N_{2}^{2} \theta_{2}=-\frac{E_{c} P_{r}}{2}\left[\left(\frac{\partial u_{1}}{\partial z}\right)^{2}-\frac{k \sigma^{2} \lambda_{1}}{\left(1+i \sigma \lambda_{1}\right)^{2}} u_{1}^{2}\right] \\
\theta_{p 2}=\frac{\theta_{2}}{1+2 i \sigma \lambda_{2}}, \quad \theta_{2}=(0,0) \quad \text { at } z=(0,1), \\
N_{2}^{2}=\frac{i \sigma\left(1+k+2 i \sigma \lambda_{2}\right)}{1+2 i \sigma \lambda_{2}} .
\end{gathered}
$$


2502 On heat transfer to pulsatile flow of a two-phase fluid

Solving (4.5) with (4.6), the steady temperature field for the fluid and the particles are given by

$$
\begin{aligned}
& \theta_{0}(z)=C+D z-\frac{E_{c} P_{r}}{24}\left(3 z^{2}-4 z^{3}+2 z^{4}\right) \\
& -\frac{E_{c} P_{r} \epsilon^{2}}{8 s_{1} s_{2} s_{3}^{2}}\left[m_{i}^{2} \cosh 2 m_{r}(1-z)-m_{r}^{2} \cos 2 m_{i}(1-z)\right. \\
& +2 m_{r}^{2} \cosh m_{r} \cos m_{i}(1-2 z)-2 m_{i}^{2} \cos m_{i} \cosh m_{r}(1-2 z) \\
& \left.+m_{i}^{2} \cosh 2 m_{r} z-m_{r}^{2} \cos 2 m_{i} z\right] \\
& -\frac{E_{c} P_{r} k \sigma^{2} \lambda_{1} \epsilon^{2}}{2\left(1+\sigma^{2} \lambda_{1}^{2}\right)}\left[\frac{z^{2}}{2 s_{1}^{2}}-\frac{2 s_{4}}{s_{1}^{4} s_{2}}\left\{\cosh m_{r}(2-z) \cos m_{i} z-\cosh m_{r} z \cos m_{i}(2-z)\right.\right. \\
& +\cosh m_{r}(1+z) \cos m_{i}(1-z) \\
& \left.-\cosh m_{r}(1-z) \cos m_{i}(1+z)\right\} \\
& +\frac{4 S_{3}}{s_{1}^{4} s_{2}}\left\{\sin m_{i} z \sinh m_{r}(2-z)-\sinh m_{r} z \sin m_{i}(2-z)\right. \\
& +\sinh m_{r}(1+z) \sin m_{i}(1-z) \\
& \left.-\sinh m_{r}(1-z) \sin m_{i}(1+z)\right\} \\
& +\frac{1}{4 s_{1}^{2} s_{2} s_{3}^{2}}\left\{m_{i}^{2} \cosh 2 m_{r}(1-z)+m_{r}^{2} \cos 2 m_{i}(1-z)\right. \\
& -2 m_{r}^{2} \cosh m_{r} \cos m_{i}(1-2 z)+m_{i}^{2} \cosh 2 m_{r} z \\
& \left.\left.-2 m_{i}^{2} \cosh m_{r}(1-2 z) \cos m_{i}+m_{r}^{2} \cos 2 m_{i} z\right\}\right] \text {, }
\end{aligned}
$$

where

$$
\begin{gathered}
\theta_{p 0}(z)=\theta_{0}(z), \\
C=\frac{E_{c} P_{r} \epsilon^{2}}{8 s_{1} s_{2} s_{3}^{2}}\left[m_{i}^{2} \cosh 2 m_{r}-m_{r}^{2} \cos 2 m_{i}+2 s_{4} \cosh m_{r} \cos m_{i}-s_{4}\right] \\
-\frac{E_{c} P_{r} k \sigma^{2} \lambda_{1} \epsilon^{2}}{2\left(1+\lambda_{1}^{2} \sigma^{2}\right)}\left[\frac{2 s_{4}}{s_{1}^{4}}-\frac{1}{4 s_{1}^{2} s_{2} s_{3}^{2}}\left(m_{i}^{2} \cosh 2 m_{r}+m_{r}^{2} \cos 2 m_{i}\right.\right. \\
\left.\left.-2 s_{1} \cosh m_{r} \cos m_{i}+s_{1}\right)\right], \\
D=1+\frac{E_{c} P_{r}}{24}+\frac{E_{c} P_{r} k \sigma^{2} \lambda_{1} \epsilon^{2}}{4 s_{1}^{2}\left(1+\sigma^{2} \lambda_{1}^{2}\right)}, \\
s_{1}=m_{r}^{2}+m_{i}^{2} \quad \cosh 2 m_{r}-\cos 2 m_{i} \quad s_{3}=m_{r} m_{i}, \quad s_{4}=m_{r}^{2}-m_{i}^{2} .
\end{gathered}
$$


In particular, when $k=0$, the steady temperature for clean fluid becomes

$$
\begin{aligned}
\theta_{0 c}= & z+\frac{E_{c} P_{r}}{24}\left(1-3 z+4 z^{2}-2 z^{3}\right) z \\
& +\frac{E_{c} P_{r} \epsilon^{2}}{8 \sigma}\left[1-\frac{\cosh \sqrt{\sigma / 2}(1-2 z)+\cos \sqrt{\sigma / 2}(1-2 z)}{\cos \sqrt{\sigma / 2}+\cosh \sqrt{\sigma / 2}}\right] .
\end{aligned}
$$

The result (4.12) shows that unlike the steady velocity field, the steady temperature field is greatly influenced by both the particles and the pressure gradient fluctuations in the fluid.

From (4.7) to (4.10), the unsteady temperature fluctuations are given by

$$
\begin{gathered}
\theta_{1}(z)=L(z)-\frac{L(0)}{\sinh M}[\sinh M(1-z)+\sinh M z] \quad\left(P_{r} \neq 1\right) \\
=\frac{E_{c}(1-\cosh M) \sinh M z}{2 M^{3}(\sinh M)^{2}}-\frac{z E_{c}}{2 M^{3}}\left[\frac{\cosh M(1-z)-\cosh M z}{\sinh M}\right] \\
\\
+\frac{E_{c}\left(z-z^{2}\right)}{2 M^{2} \sinh M}[\sinh M(1-z)+\sinh M z] \quad\left(P_{r}=1\right), \\
\theta_{2}(z)=\frac{1}{\sinh \sqrt{2 P_{r} N_{2}}}\left[R(0) \sinh \sqrt{2 P_{r}} N_{2}(1-z)+R(1) \sinh \sqrt{2 P_{r}} N_{2} z\right]-R(z),
\end{gathered}
$$

where

$$
\begin{aligned}
& L(z)=\frac{E_{c} P_{r}}{M^{2}-P_{r} N_{1}^{2}}\left[\frac{1}{\sinh \sqrt{P_{r}} N_{1}}\left(\frac{\cosh M-1}{M \sinh M}-\frac{4}{M^{2}-P_{r} N_{1}^{2}}\right)\right. \\
& \times\left(\sinh \sqrt{P_{r}} N_{1} z+\sinh \sqrt{P_{r}} N_{1}(1-z)\right) \\
& \left.+\frac{1-2 z}{M \sinh M}\{\cosh M z-\cosh M(1-z)\}\right], \\
& L(0)=L(1)=-\frac{4 E_{c} P_{r}}{\left(M^{2}-P_{r} N_{1}^{2}\right)^{2}}, \\
& R(z)=\frac{E_{c} P_{r}}{4 M^{2} \cosh ^{2}(M / 2)}\left[\frac{\cosh M(1-2 z)}{4 M^{2}-2 P_{r} N_{2}^{2}}+\frac{1}{2 P_{r} N_{2}^{2}}\right] \\
& +\frac{E_{c} P_{r} \delta^{2}}{2 M^{4}}\left[\frac{1}{2 P_{r} N_{2}^{2}}\left(1+\frac{1}{2 \cosh ^{2}(M / 2)}\right)+\frac{2}{M^{2}-2 P_{r} N_{2}^{2}}\right. \\
& \left.\times \frac{\cosh (1 / 2) M(1-2 z)}{\cosh (M / 2)}-\frac{\cosh M(1-2 z)}{4 \cosh ^{2}(M / 2)\left(2 M^{2}-P_{r} N_{2}^{2}\right)}\right],
\end{aligned}
$$


2504 On heat transfer to pulsatile flow of a two-phase fluid

$$
\begin{aligned}
& R(0)=R(1)=\frac{E_{c} P_{r}}{4 M^{2} \cosh ^{2}(M / 2)} {\left[\frac{\cosh M}{4 M^{2}-2 P_{r} N_{2}^{2}}+\frac{1}{2 P_{r} N_{2}^{2}}\right] } \\
&+\frac{E_{c} P_{r} \delta^{2}}{2 M^{4}} {\left[\frac{1}{2 P_{r} N_{2}^{2}}\left(1+\frac{1}{\cosh ^{2}(M / 2)}\right)+\frac{2}{M^{2}-2 P_{r} N_{2}^{2}}\right.} \\
&\left.-\frac{\cosh M}{4 \cosh ^{2}(M / 2)\left(2 M^{2}-P_{r} N_{2}^{2}\right)}\right], \\
& \delta^{2}=\frac{k \sigma^{2} \lambda_{1}}{\left(1+i \sigma \lambda_{1}\right)^{2}} .
\end{aligned}
$$

In the limit $k \rightarrow 0, M^{2}=N_{1}^{2}=N_{2}^{2}=i \sigma$. So the results for $\theta_{1}$ and $\theta_{2}$ can be derived easily for the case of clean fluid. The corresponding results for the temperature fluctuations of the particles are

$$
\theta_{p 1}=\frac{\theta_{1}}{1+i \sigma \lambda_{2}}, \quad \theta_{p 2}=\frac{\theta_{2}}{1+2 i \sigma \lambda_{2}}
$$

\section{Rate of heat transfer}

The rate of heat transfer per unit area at the plate $z=0$ is given by

$$
\begin{aligned}
Q_{0}=\left(\frac{\partial \theta}{\partial z}\right)_{z=0} & =\left(\frac{\partial \theta_{0}}{\partial z}\right)_{z=0}+\epsilon e^{i \sigma t}\left(\frac{\partial \theta_{1}}{\partial z}\right)_{z=0}+\epsilon^{2} e^{2 i \sigma t}\left(\frac{\partial \theta_{2}}{\partial z}\right)_{z=0} \\
& =\left(\theta_{0}^{\prime}\right)_{z=0}+\epsilon\left|D_{0}\right| \cos \left(\sigma t+\alpha_{0}\right)+\epsilon^{2}\left|D_{1}\right| \cos \left(2 \sigma t+\alpha_{1}\right),
\end{aligned}
$$

where

$$
\begin{aligned}
& \left(\frac{\partial \theta_{0}}{\partial z}\right)_{z=0}=1+\frac{E_{c} P_{r}}{24}+\frac{E_{c} P_{r} \epsilon^{2}}{4 s_{1}\left(\cosh m_{r}+\cos m_{i}\right)}\left(\frac{\sinh m_{r}}{m_{r}}-\frac{\sin m_{i}}{m_{i}}\right) \\
& +\frac{E_{c} P_{r} k \sigma^{2} \lambda_{1} \epsilon^{2}}{4 s_{1}^{2}\left(1+\sigma^{2} \lambda_{1}^{2}\right)}\left[1-\frac{m_{i}\left(3 m_{r}^{2}-m_{i}^{2}\right) \sinh m_{r}-m_{r}\left(m_{r}^{2}-3 m_{i}^{2}\right) \sin m_{i}}{s_{1} s_{3}\left(\cosh m_{r}+\cos m_{i}\right)}\right], \\
& \left(\frac{\partial \theta_{1}}{\partial z}\right)_{z=0}=\frac{E_{c} P_{r}}{M^{2}-P_{r} N_{1}^{2}}\left[\frac{\sqrt{P_{r}} N_{1}}{\sinh \sqrt{P_{r}} N_{1}}\left(\frac{\cosh M-1}{M \sinh M}-\frac{4}{M^{2}-P_{r} N_{1}^{2}}\right)\right. \\
& \times\left(1-\cosh \sqrt{P_{r}} N_{1}\right)-\frac{2(1-\cosh M)}{M \sinh M} \\
& \left.+\frac{4 M(1-\cosh M)}{\left(M^{2}-P_{r} N_{1}^{2}\right) \sinh M}+1\right], \quad P_{r} \neq 1, \\
& \left(\frac{\partial \theta_{2}}{\partial z}\right)_{z=0}=\frac{\sqrt{2 P_{r}} N_{2}}{\sinh \sqrt{2 P_{r}} N_{2}}\left\{R(1)-R(0) \cosh \sqrt{2 P_{r}} N_{2}\right\} \\
& +\frac{3 E_{c} P_{r} \delta^{2}}{2 M\left(2 M^{2}-P_{r} N_{2}^{2}\right)\left(M^{2}-2 P_{r} N_{2}^{2}\right)} \frac{\sinh (M / 2)}{\cosh (M / 2)} \\
& +\frac{E_{c} P_{r}}{2 M\left(2 M^{2}-P_{r} N_{2}^{2}\right)} \frac{\sinh (M / 2)}{\cosh (M / 2)},
\end{aligned}
$$


where

$$
\begin{aligned}
& D_{0}=D_{0 r}+i D_{0 i}, \quad \tan \alpha_{0}=\frac{D_{0 i}}{D_{0 r}} \\
& D_{1}=D_{1 r}+i D_{1 i}, \quad \tan \alpha_{1}=\frac{D_{1 i}}{D_{1 r}}
\end{aligned}
$$

The expression for $\left(\partial \theta_{0} / \partial z\right)_{z=0}$ shows that the presence of particles $(k \neq 0)$ increases or decreases the rate of heat transfer in the steady state condition at the lower wall if the quantity within the third bracket is positive or negative. On the other hand, in absence of particles $(k=0)$, the rate of heat transfer in the steady situation at the lower wall becomes

$$
Q_{0 c}=1+\frac{E_{c} P_{r}}{24}+\frac{E_{c} P_{r} \epsilon^{2}}{(2 \sigma)^{3 / 2}} F(\sigma)
$$

which is always positive where $F(\sigma)=(\sinh \sqrt{\sigma / 2}-\sin \sqrt{\sigma / 2}) /(\cosh \sqrt{\sigma / 2}+\cos \sqrt{\sigma / 2})$.

Similarly, the rate of heat transfer per unit area at the upper wall $z=1$ is given by

$$
\begin{aligned}
& Q_{1}=\left(\frac{\partial \theta}{\partial z}\right)_{z=1}=\left(\frac{\partial \theta_{0}}{\partial z}\right)_{z=1}+\epsilon e^{i \sigma t}\left(\frac{\partial \theta_{1}}{\partial z}\right)_{z=1}+\epsilon^{2} e^{2 i \sigma t}\left(\frac{\partial \theta_{2}}{\partial z}\right)_{z=1} \\
& =1-\frac{E_{c} P_{r}}{24}-\frac{E_{c} P_{r} \epsilon^{2}}{4 s_{1}\left(\cosh m_{r}+\cos m_{i}\right)}\left[\frac{\sinh m_{r}}{m_{r}}-\frac{\sin m_{i}}{m_{i}}\right] \\
& -\frac{E_{c} P_{r} k \sigma^{2} \lambda_{1} \epsilon^{2}}{4 s_{1}^{2}\left(1+\sigma^{2}\right)}\left[1-\frac{m_{i}\left(3 m_{r}^{2}-m_{i}^{2}\right) \sinh m_{r}-m_{r}\left(m_{r}^{2}-3 m_{i}^{2}\right) \sin m_{i}}{s_{1} s_{3}\left(\cosh m_{r}+\cos m_{i}\right)}\right] \\
& -\epsilon e^{i \sigma t} \frac{E_{c} P_{r}}{M^{2}-P_{r} N_{1}^{2}}\left[\frac{\sqrt{ } P_{r} N_{1}}{\sinh \sqrt{ } P_{r} N_{1}}\left(\frac{\cosh M-1}{M \sinh M}-\frac{4}{M^{2}-P_{r} N_{1}^{2}}\right)\right. \\
& \times\left(1-\cosh \sqrt{ } P_{r} N_{1}\right)+\frac{4 M(1-\cosh M)}{\left(M^{2}-P_{r} N_{1}^{2}\right) \sinh M} \\
& \left.-\frac{2(1-\cosh M)}{M \sinh M}+1\right] \\
& -\epsilon^{2} e^{2 i \sigma t}\left[\frac{\sqrt{2 P_{r}} N_{2}}{\sinh \sqrt{2 P_{r}} N_{2}}\left\{R(1)-R(0) \cosh \sqrt{2 P_{r}} N_{2}\right\}\right. \\
& -\frac{E_{c} P_{r} \delta^{2}}{M^{3}} \tanh \frac{M}{2}\left\{\frac{1}{2\left(2 M^{2}-P_{r} N_{2}^{2}\right)}-\frac{1}{M^{2}-2 P_{r} N_{2}^{2}}\right\} \\
& \left.+\frac{E_{c} P_{r}}{2 M} \tanh \frac{M}{2} \frac{1}{\left(2 M^{2}-P_{r} N_{2}^{2}\right)}\right] \\
& =\left(\theta_{0}^{\prime}\right)_{z=1}-\epsilon\left|D_{0}\right| \cos \left(\sigma t+\alpha_{0}\right)-\epsilon^{2}\left|D_{1}\right| \cos \left(2 \sigma t+\alpha_{1}\right) \text {. }
\end{aligned}
$$




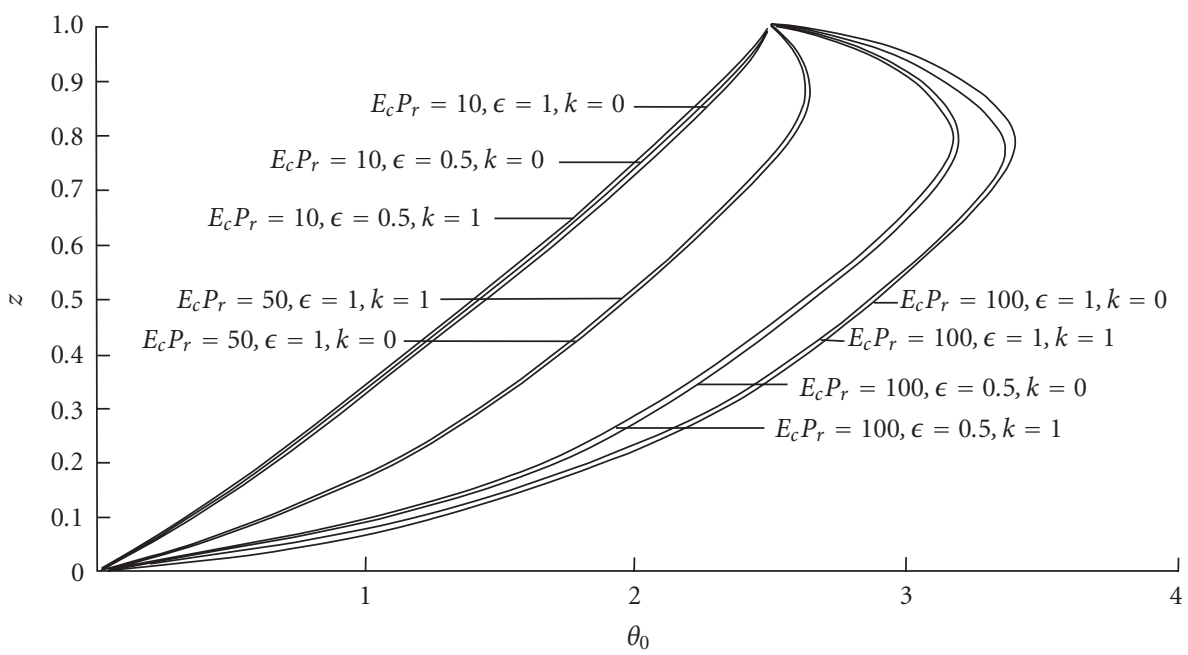

Figure 6.1. Steady temperature profiles in a two-phase fluid.

The rate of heat transfer per unit area for the clean fluid at the upper wall $z=1$ is given by

$$
Q_{1 c}=1-\frac{E_{c} P_{r}}{24}-\frac{E_{c} P_{r} \epsilon^{2}}{(2 \sigma)^{3 / 2}} F(\sigma) .
$$

\section{Numerical results and discussions}

For the problem under investigation, $\theta_{0}$ represents the steady temperature distribution in the two-phase fluid-particle system. The expression for $\theta_{0}$ contains one linear term corresponding to the fluid at rest, a biquadratic term which arises due to viscous friction and a term involving $\epsilon^{2}$ which corresponds to the mean heating of the fluid due to dissipation of energy caused by the pressure gradient fluctuations. It is interesting to note that the effect of the particles modifies the mean heating of the fluid only when the pressure gradient fluctuates. Hence the expression for $\theta_{0}$ is not the same for both viscous and particulate fluids. The temperature profiles corresponding to $\theta_{0}$ are shown in Figure 6.1 for various values of $E_{c} P_{r}, k$, and $\epsilon$. The graphical representation clearly indicates that the value of $\theta_{0}$ increases with both $E_{c} P_{r}$ and $\epsilon$ but decreases with the increase of particle concentration $(k)$. Moreover, in all cases, the maximum value of $\theta_{0}$ occurs near the boundary layer of the hotter wall.

Regarding the rate of heat transfer in the steady-state condition, the reversal of heat flux from the fluid to the hotter wall takes place when $E_{c} P_{r}$ exceeds a critical value depending on $\epsilon$ and $k$ which in turn makes the hotter wall more hot. For example, when $\epsilon=0, k=0$, heat flows from the fluid to the hotter wall when $E_{c} P_{r}>24$. This case corresponds to the heat transfer in a clean fluid under constant pressure gradient when 
Table 6.1. Critical values of $E_{c} P_{r}$ for the reversal of heat flux at the hotter wall when $\sigma=10, \lambda_{1}=0.1$.

\begin{tabular}{lccc}
\hline$k / \epsilon$ & 0 & 0.5 & 1.0 \\
\hline 0 & 24 & 22.5 & 19.1 \\
0.1 & 24 & 22.5 & 19.1 \\
0.6 & 24 & 22.6 & 19.2 \\
0.7 & 24 & 22.6 & 19.3 \\
1.0 & 24 & 22.7 & 19.5 \\
\hline
\end{tabular}

the walls are maintained at constant temperatures $T_{0}$ and $T_{1}\left(>T_{0}\right)$. On the other hand, for $\epsilon=0.5$ and $k=0.1$, the reversal of heat flux at the hotter wall takes place when $E_{c} P_{r}>22.5$ which is further enhanced with the increase of $k$. Alternately, when $\epsilon=1.0$ and $k=0.1$, the reversal of heat flux from fluid to the hotter wall takes place when $E_{c} P_{r}>19.1$. All these results are shown in Table 6.1, and on the basis of these results we conclude that the critical value of $E_{c} P_{r}$ responsible for the reversal of heat flux from the fluid to the hotter wall diminishes with the increase of $\epsilon$ and increases with the increase of particle concentration in the fluid. In fact, the value of $E_{c} P_{r}$ provides a measure of the amount of heat generated due to friction, which, in the present case, increases with the increase of the pressure gradient. As a result, if the temperature difference between the walls is fixed, heat flows from the hotter wall to the fluid as long as the pressure gradient does not exceed a certain value depending on the amount of fluctuations and the presence of the particles. This phenomenon is important for cooling at high pressure gradient. However, if instead of pressure gradient, the motion of the fluid is produced otherwise, such as in the case of steady Couette flow of viscous or two-phase fluids under a constant pressure gradient, the critical value of $E_{c} P_{r}$ for the reversal of heat flux at the hotter wall is found as 2. Such a reversal of heat flux occurs only when the motion of the upper (hotter) wall exceeds certain velocity provided the temperature difference between the walls remains constant.This phenomenon is also important for cooling at high velocity and is reported by Schlichting [5]. We therefore conclude that the critical value of $E_{c} P_{r}$ for the reversal of heat flux at the hotter wall is much higher in the case of cooling at high pressure gradient compared to its value for cooling at high velocity. The effect of Eckert number $E_{c}$ on the steady heat transfer coefficient for various values of pressure gradient fluctuation $\epsilon$ and the particle concentration $k$ is shown in the Table 6.2.

The instantaneous temperature profiles are plotted in Figures 6.2, 6.3, and 6.4. Figure 6.2 exhibits the instantaneous temperature profiles for viscous and particulate fluids for different values of $\sigma t$ when $k=0$ and $0.3, E_{c} P_{r}=100, \lambda_{1}=0.1, \lambda_{2}=0.3$. It is to be noted here that the results for $k=0$ always represents the case of a viscous fluid irrespective of the values of $\lambda_{1}$ and $\lambda_{2}$. Moreover, it is evident from Figure 6.2 that the presence of particles diminishes the temperature near the walls and increases the same at the central part of the channel. Figure 6.3 presents the instantaneous temperature profiles in three cases corresponding to the values of $\lambda_{1}<=>\lambda_{2}$ when $k, E_{c} P_{r}$, and $\sigma t$ are fixed while the Figure 6.4 provides the unsteady temperature profiles for different values of $\sigma t$ when $E_{c} P_{r}$ is as large as 300 . Finally, we notice that the temperature fluctuations increase the rate of 
2508 On heat transfer to pulsatile flow of a two-phase fluid

Table 6.2. Steady heat transfer coefficient $\left(\theta_{0}^{\prime}\right)_{z=0}$ and $\left(\theta_{0}^{\prime}\right)_{z=1}$ for $\sigma=10, \lambda_{1}=0.1, P_{r}=10$.

\begin{tabular}{|c|c|c|c|c|c|c|}
\hline & $\epsilon$ & $k / E_{c}$ & 1 & 2 & 3 & 5 \\
\hline \multirow{6}{*}{$\left(\theta_{0}^{\prime}\right)_{z=0}$} & 0.0 & 0.0 & 1.41667 & 1.8333 & 2.25 & 3.08333 \\
\hline & & 0.5 & 1.41667 & 1.8333 & 2.25 & 3.08333 \\
\hline & 0.5 & 0.0 & 1.44274 & 1.88548 & 2.32822 & 3.21370 \\
\hline & & 0.5 & 1.43765 & 1.87531 & 2.31296 & 3.18827 \\
\hline & 1.0 & 0.0 & 1.52096 & 2.04192 & 2.56288 & 3.60480 \\
\hline & & 0.5 & 1.50061 & 2.00123 & 2.50184 & 3.50307 \\
\hline \multirow{6}{*}{$\left(\theta_{0}^{\prime}\right)_{z=1}$} & 0.0 & 0.0 & 0.58333 & 0.16667 & -0.25 & -1.08333 \\
\hline & & 0.5 & 0.58333 & 0.16667 & -0.25 & -1.08333 \\
\hline & 0.5 & 0.0 & 0.55726 & 0.11452 & -0.32822 & -1.21370 \\
\hline & & 0.5 & 0.56716 & 0.12534 & -0.32415 & -1.21042 \\
\hline & 1.0 & 0.0 & 0.47904 & -0.04162 & -0.56288 & -1.60480 \\
\hline & & 0.5 & 0.48867 & -0.03265 & -0.56098 & -1.60163 \\
\hline
\end{tabular}

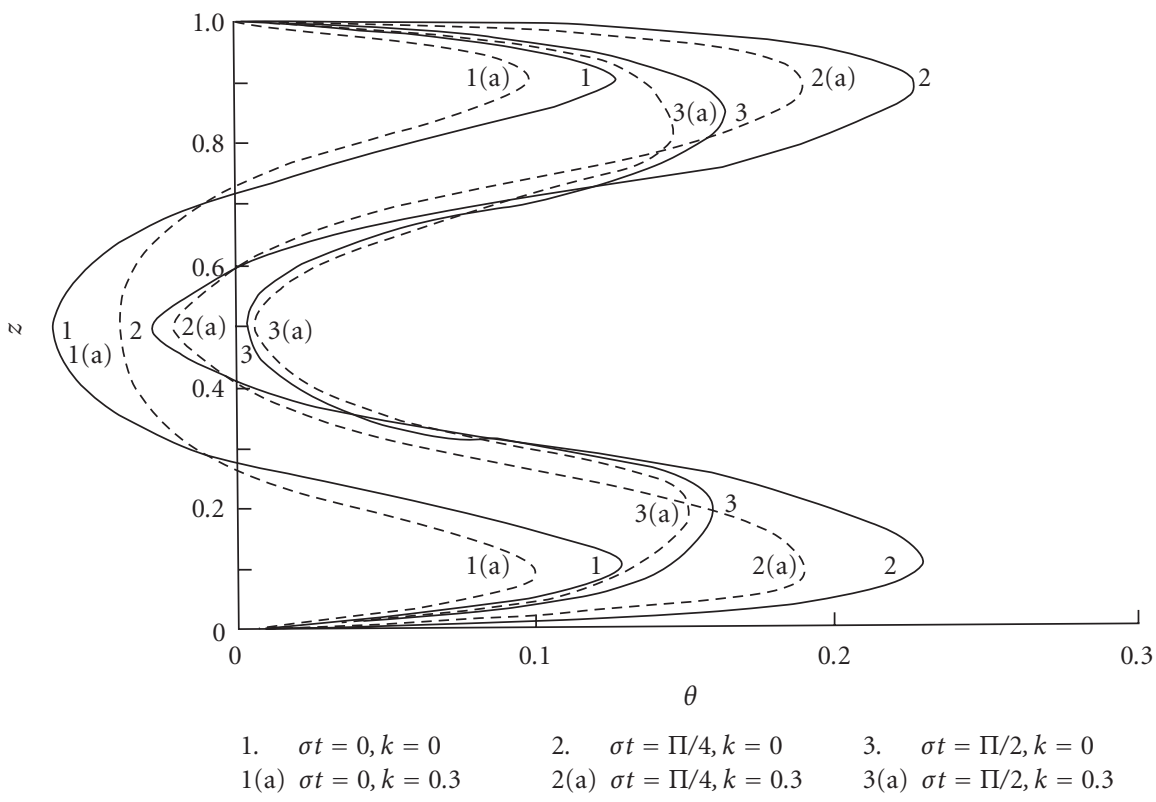

Figure 6.2. Effect of particle concentration $(k)$ on the unsteady temperature profiles in a two-phase fluid when $E_{c} P_{r}=100 . \lambda_{1}=0.1, \lambda_{2}=0.3$.

heat transfer at the colder wall and decrease the same at the hotter wall irrespective of the influences of other flow parameters. This phenomenon is evident from the results (5.1) and (5.5). 


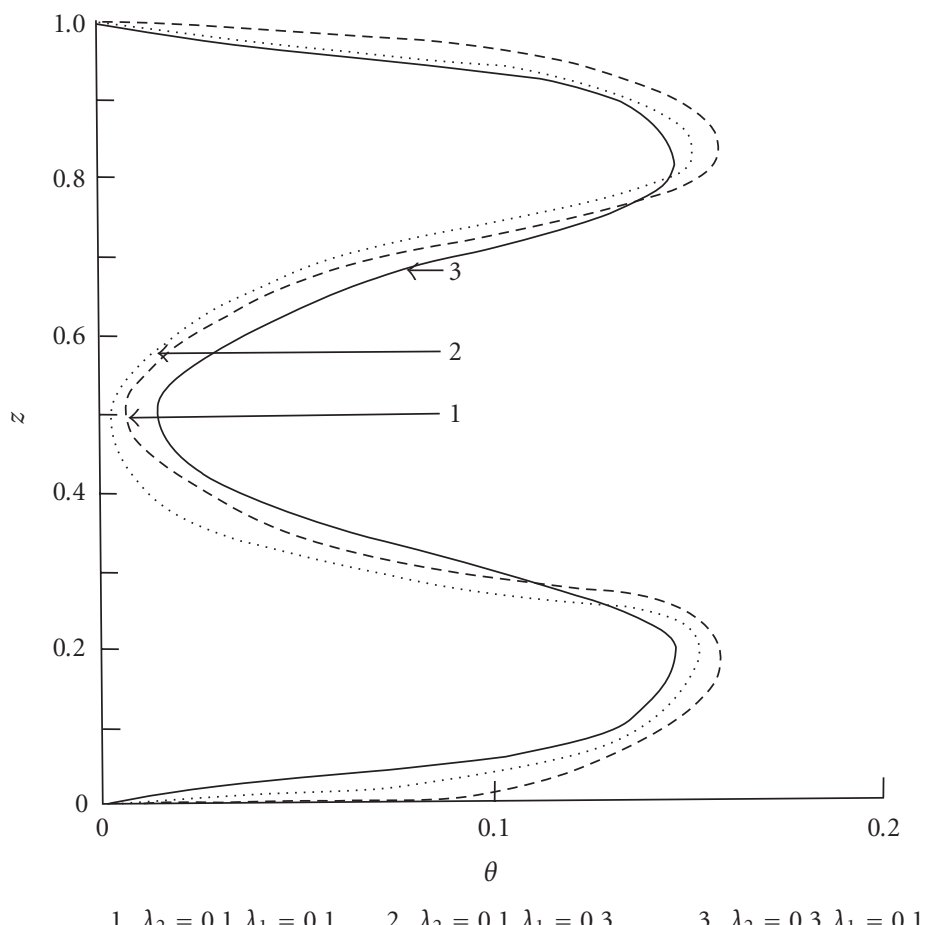

Figure 6.3. Effect of velocity relaxation time $\left(\lambda_{1}\right)$ and thermal relaxation time $\left(\lambda_{2}\right)$ on unsteady temperature profiles in a two-phase fluid when $\sigma t=\Pi / 2, k=0.3, E_{c} P_{r}=100$.

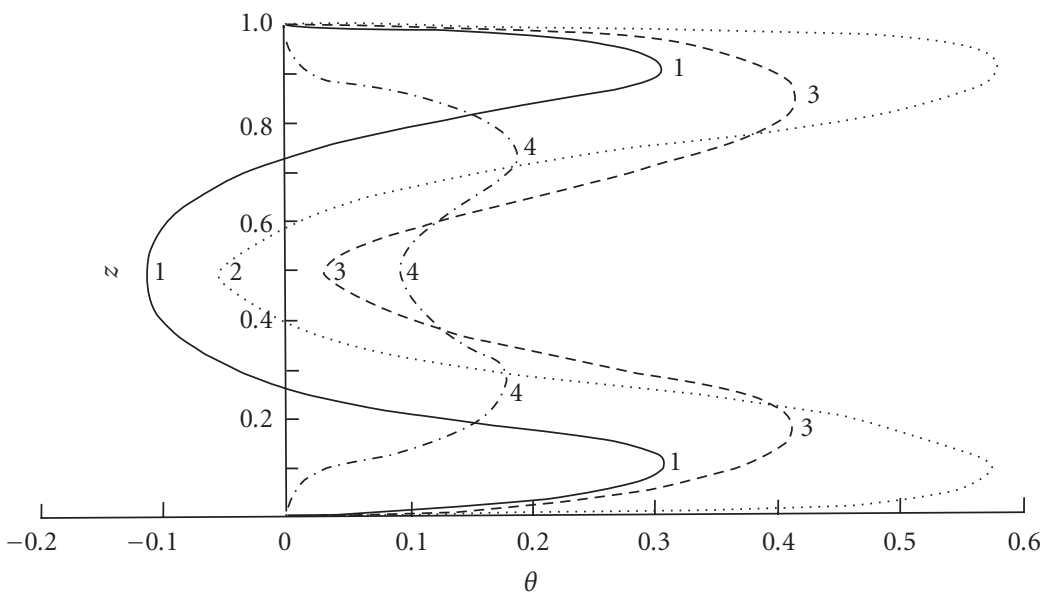
1. $\sigma t=0$
3. $\sigma t=\Pi / 2$
2. $\sigma t=\Pi / 4$
4. $\sigma t=3 \Pi / 4$

Figure 6.4. Unsteady temperature profiles in two-phase fluid when $E_{c} P_{r}=300, k=0.3, \lambda_{1}=0.1, \lambda_{2}=$ 0.3 . 
2510 On heat transfer to pulsatile flow of a two-phase fluid

\section{References}

[1] A. K. Ghosh and L. Debnath, On heat transfer to pulsatile flow of a viscoelastic fluid, Acta Mech. 93 (1992), no. 1-4, 169-177.

[2] F. E. Marble, Dynamics of dusty gases, Annu. Rev. Fluid Mech. 2 (1970), 397-446.

[3] G. Radhakrishnamacharya and M. K. Maiti, Heat transfer to pulsatile flow in a porous channel, Int. J. Heat Mass Transfer 20 (1977), no. 2, 171-173.

[4] P. G. Saffman, On the stability of laminar flow of a dusty gas, J. Fluid Mech. 13 (1962), 120-128.

[5] H. Schlichting, Boundary Layer Theory, 6th ed., McGraw-Hill, New York, 1968.

A. K. Ghosh: Department of Mathematics, Jadavpur University, Calcutta-700032, India E-mail address: akg10143@yahoo.co.in

S. P. Chakraborty: Department of Mathematics, Behala College, Calcutta-700060, India 


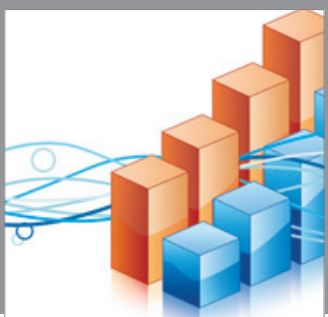

Advances in

Operations Research

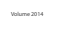

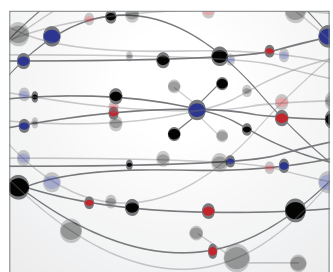

\section{The Scientific} World Journal
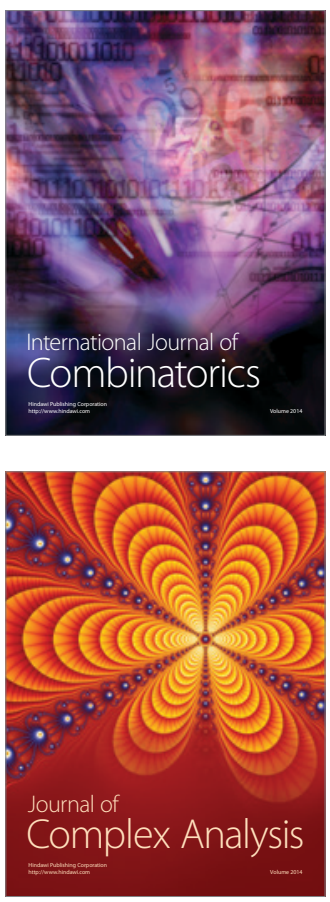

International Journal of

Mathematics and

Mathematical

Sciences
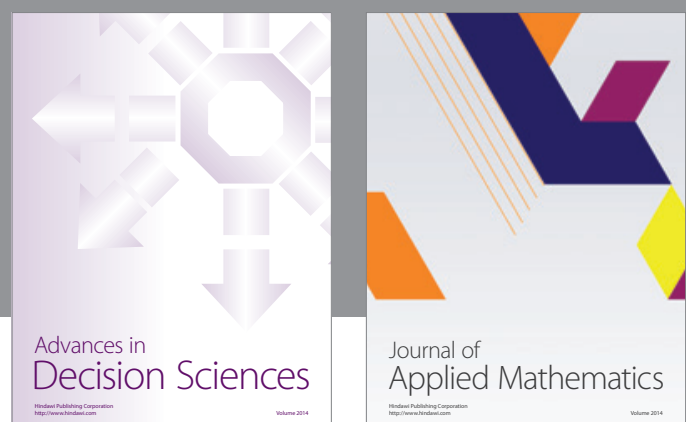

Journal of

Applied Mathematics
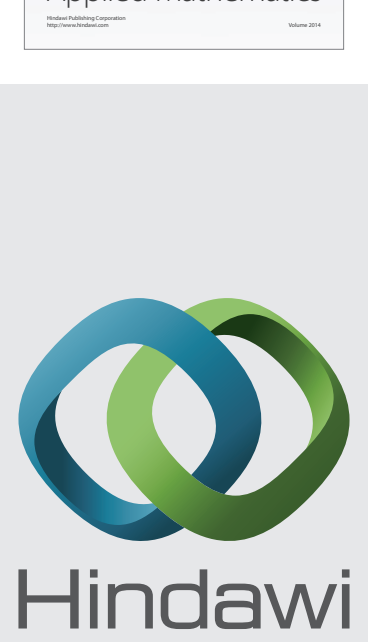

Submit your manuscripts at http://www.hindawi.com
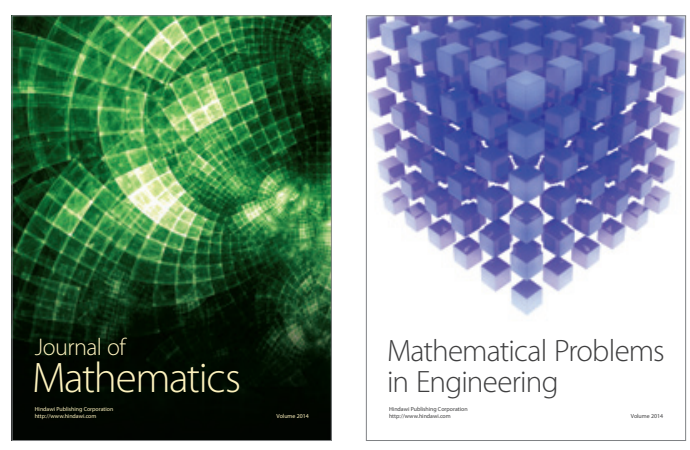

Mathematical Problems in Engineering
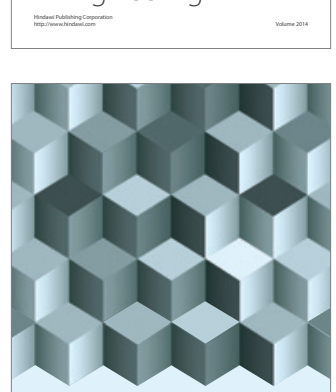

Journal of

Function Spaces
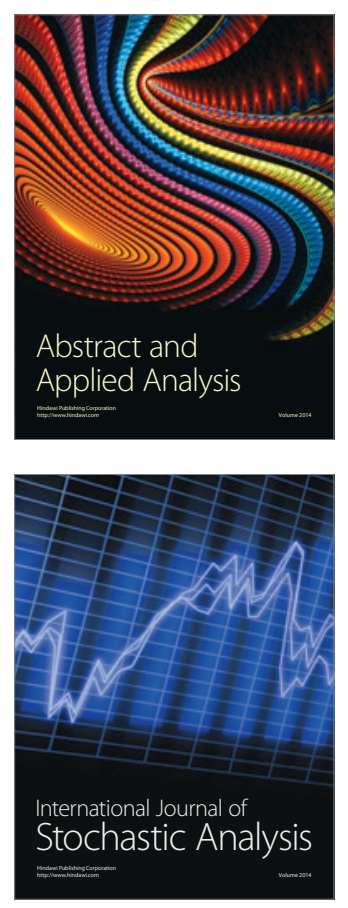

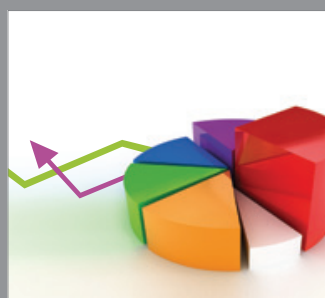

ournal of

Probability and Statistics

Promensencen
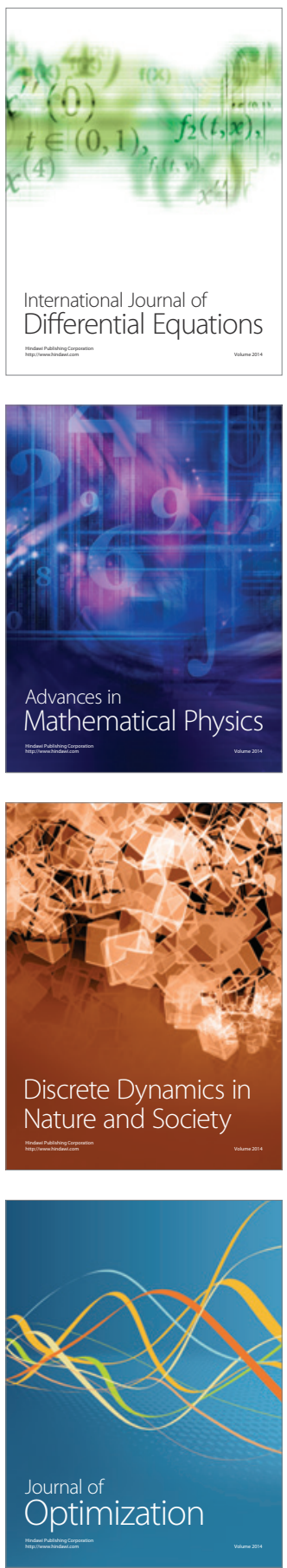